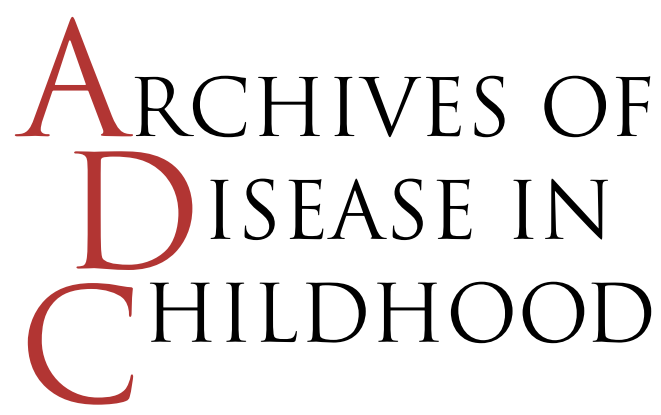

\title{
Meconium aspiration syndrome and extracorporeal membrane oxygenation
}

Meconium aspiration syndrome (MAS) is a common cause of lung disease in neonates. Meconium staining of amniotic fluid occurs in around $10 \%$ of all deliveries at term. Asphyxia before birth stimulates intestinal peristalsis and relaxation of the anal sphincter, and in more severe cases, gasping in utero, leading to meconium aspiration. Respiration after birth draws meconium firstly into the major airways and subsequently into the smaller airways, where it may cause obstruction, atelectasis, air trapping, and pneumothorax. Meconium can also cause a chemical pneumonitis and inactivation of surfactant, further impairing gas exchange and potentiating barotrauma. Infants with severe MAS rapidly develop pulmonary hypertension with right to left shunting through a patent foramen ovale and at the level of the arterial duct; this shunting further potentiates hypoxia. Severe MAS occurs in around 1 in 500 births, ${ }^{1}$ and is characterised by profound hypoxaemia, where the coexistence of parenchymal lung damage and severe pulmonary hypertension can result in a vicious cycle of clinical decline, which is often simply exacerbated by the necessary escalation of "conventional" treatment.

As the field of perinatology has advanced, much attention has been focused on the prevention of MAS, with emphasis on timely delivery and skilled neonatal resuscitation. In infants born with meconium staining of the liquor, the oropharynx should be suctioned before they take their first breath, preferably while still at the perineum. ${ }^{2}$ However, absence of meconium from the oropharynx does not necessarily preclude aspiration distal to the vocal cords, ${ }^{3}$ and routine intubation with endotracheal toilet does not always prevent the subsequent development of MAS. ${ }^{4}$ Therefore despite the best efforts of those resuscitating a meconium affected neonate, a number of these babies go on to develop MAS, with acute respiratory failure requiring positive pressure ventilation.

MAS carries with it not only significant mortalityestimated in one study to be as high as 1 in 12 cases requiring mechanical ventilation ${ }^{1}$ - but also major morbidity. Chronic lung disease can result from both the pulmonary effects of meconium itself and the ventilator induced lung injury, which is often regarded as an "inevitable consequence" of the levels of mechanical ventilation that these neonates receive. Furthermore, the extrapulmonary effects of prolonged severe hypoxia, in particular on the brain, and the significant incidence of neurological injury should not be underestimated in this vulnerable group of infants.

Various ventilator strategies have been suggested for neonates with MAS, particularly to control pulmonary hypertension and provide protection from pneumothorax. Inhaled nitric oxide, a selective pulmonary vasodilator, is typically administered to these infants when available, although previous studies have suggested that it may be less effective in the subgroup with MAS. ${ }^{5}$ This may in part be due to the physical "barrier" to its diffusion across the alveolar membrane which is produced by the presence of meconium in the distal airways.

Thus, despite these measures, a small subgroup of patients with MAS go on to develop intractable pulmonary hypertension with profound hypoxia, co-existent with a variable degree of parenchymal lung injury. In these infants, the evidence would suggest that extracorporeal membrane oxygenation (ECMO) is the most effective mode of treatment. The natural history of pulmonary hypertension is, in most cases, spontaneous recovery with time. The institution of ECMO therefore provides global tissue oxygenation while allowing pulmonary vasculature and lung parenchyma time to recover from the antecedent insults.

\section{ECMO in the treatment of MAS}

The use of ECMO in the treatment of MAS was first reported by Bartlett et al in 1977, ${ }^{6}$ who used ECMO to treat eight moribund neonates. Three of these infants survived, as compared with a $90 \%$ mortality in a "conventionally" treated group. The use of venoarterial (V-A) ECMO became increasingly popular throughout the United States during the 1980s, with consistently encouraging results for neonates with severe $\mathrm{MAS}^{7}$ The criteria for instituting ECMO support were also refined over this time, and an oxygenation index of greater than 40 was suggested as a referral criterion. ${ }^{8}$ The oxygenation index is calculated as follows:

Oxygenation index $=\mathrm{Paw} \times \mathrm{FIO}_{2} / \mathrm{PaO}_{2}$

where $\mathrm{Paw}=$ mean airway pressure, $\mathrm{FIO}_{2}=$ inspired oxygen fraction $\times 100$, and $\mathrm{PaO}_{2}=$ arterial oxygen tension (mm Hg).

Thus by the early 1990s, data from the extracorporeal life support organisation ${ }^{9}$ and three small randomised trials in the United States ${ }^{10-13}$ strongly supported the important 
role of ECMO in the management of neonatal respiratory failure. However, it was only after the publication of the UK collaborative neonatal ECMO trial $^{14}$ that ECMO became a relatively accepted method of support for neonates with MAS in this country. The results of this trial, which again used an oxygenation index of 40 as a referral criterion, concluded that, for every four infants receiving ECMO for MAS, there was one extra survivor. Furthermore, although infants with MAS tend to do relatively less well when treated conventionally than other term neonates with respiratory failure, the converse is true when ECMO support is used. The survival figures for neonates with MAS who receive ECMO are extremely encouraging, and UK ECMO centres currently quote survival figures of around $90 \%$ for neonates in whom the primary indication is pulmonary hypertension, without associated severe lung injury attributable to prolonged aggressive ventilation.

\section{ECMO techniques}

Two modes of ECMO can be used to support neonates with MAS. Most ECMO centres initially used V-A support, in which the right common carotid artery and internal jugular veins were cannulated, thus providing cardiac and respiratory support. More recently, however, venovenous $(\mathrm{V}-\mathrm{V})$ ECMO has emerged as the method of choice for neonates with hypoxic respiratory failure and without significant haemodynamic instability. ${ }^{15}$ In V-V ECMO, a double lumen venous cannula is inserted into the right internal jugular vein with its tip in the right atrium; one lumen carries "venous" blood from the patient to the oxygenator, and the "arterial" lumen returns oxygenated blood to the heart. ${ }^{16-18}$ Most neonates with MAS now receive V-V ECMO.

Inotropes are not a contraindication to $\mathrm{V}-\mathrm{V}$ ECMO. Modest doses of inotropes are often required to balance the detrimental cardiovascular effects of high airway pressures. Furthermore, hypoxia itself is a potent pulmonary vasoconstrictor, and thus right ventricular performance is often significantly diminished as a result of increased afterload. Finally, global hypoxia takes its toll on the myocardium, the oxygen demands of which will by far outweigh its supply, and so myocardial performance can be impaired as a result.

The potential advantages of $\mathrm{V}-\mathrm{V}$ over $\mathrm{V}-\mathrm{A}$ ECMO are the reduced risk of intracranial haemorrhage and the theoretical advantage of preserving the intact carotid circulation. Cannulation of the artery, as well as predisposing to arterial intracranial bleeds while on ECMO, will inevitably lead to disruption of flow after decannulation, either as a result of ligation of the vessel or because of the presence of a substrate for turbulence or aneurysm formation if the vessel is repaired. ${ }^{19} 20$

\section{Duration of ECMO support}

ECMO support is usually required for between 100 and 120 hours ${ }^{18}$ in MAS, the shortest duration for any neonatal diagnosis. However, this duration will inevitably be significantly increased if high pressure/volume ventilation has led to air leaks before cannulation. While on ECMO, the infant receives "resting ventilation" with slow background ventilation with a moderate level of PEEP to maintain lung expansion (rate 10/min, pressures 20/10). Serial radiographs often show a complete "white out" during the first two to three days (fig 1), with subsequent reappearance of air bronchograms and resolution of the changes as the lungs recover. Lung compliance can be estimated both manually and mechanically while the infant is on ECMO. Unless precipitous decannulation is required for other reasons - for example, large intracranial bleed - the infant is weaned from support when only minimal ventilatory support is needed to

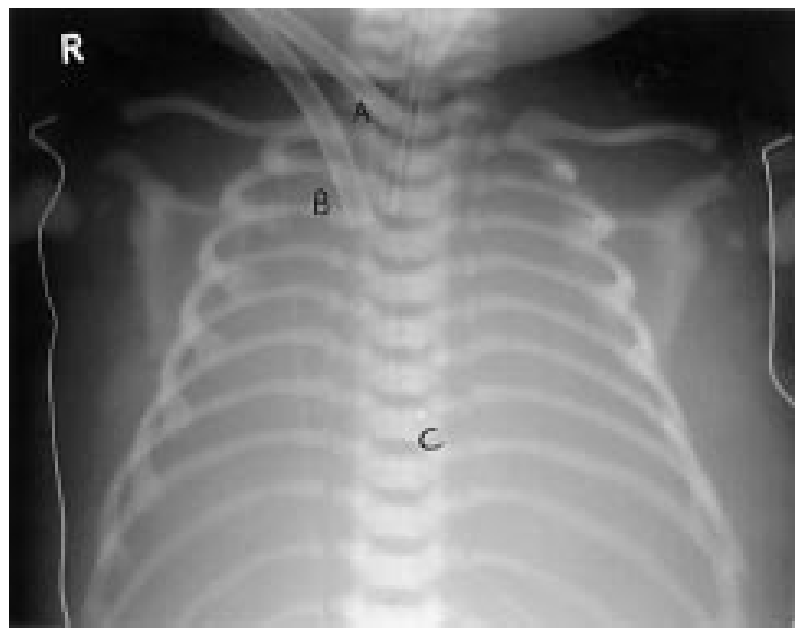

Figure 1 Chest radiograph of infant with severe meconium aspiration syndrome showing typical "white out" appearance 24 hours after institution of venoarterial extracorporeal membrane oxygenation support. The arterial cannula $(A)$ has been inserted into the right common carotid artery, with its tip in the aortic arch; the venous cannula (B) has been inserted into the right internal jugular vein and its tip $(C)$ lies in the right atrium.

\section{Key messages}

Infants with severe MAS are relatively "ideal" candidates for ECMO in that:

- almost all are $>34$ weeks gestation and weigh $>2 \mathrm{~kg}$ (cut off for ECMO)

- they have a potentially reversible respiratory problem

- associated pulmonary hypertension, although severe, is almost always reversible

- these infants need the "lung rest" that ECMO provides

- ECMO is an effective form of treatment for infants meeting the criteria

- as a subgroup, patients with MAS have a very favourable outcome

provide adequate lung expansion, oxygenation, and gas exchange. At this stage, there is usually radiological evidence of significant lung recovery.

\section{Long term outcome in MAS treated with ECMO}

The worldwide survival rate for over 5000 neonates with MAS receiving ECMO support is $94 \%,{ }^{21}$ which is in line with the UK experience when an oxygenation index of 40 is used as the referral criterion. This is the highest survival rate for any neonatal condition suitable for ECMO. There is also evidence to suggest reduced mechanical and patient related complication rates compared with other subgroups. ${ }^{22}$

In terms of long term morbidity, survivors of ECMO do not appear to have any increased rate of disability or neurological damage than other severely hypoxic, conventionally treated neonates with MAS, despite a greater proportion surviving. ${ }^{23} 24$

\section{Alternatives to ECMO in severe MAS}

Other forms of treatment have been suggested for neonates with MAS, including surfactant, ${ }^{25-27}$ inhaled nitric oxide, ${ }^{28}{ }^{29}$ and even liquid ventilation. ${ }^{30}$ However, despite some improvements in oxygenation even within these trials, there were still neonates who required ECMO support. Indeed these various forms of support and therapy are not necessarily mutually exclusive. For instance, surfactant has also been used in neonates supported with ECMO, resulting in improved pulmonary mechanics, reduced time on ECMO, and reduced disease complications. ${ }^{31}$ 


\section{Conclusions}

Despite the weight of evidence pointing to the efficacy of ECMO in supporting neonates with severe MAS, without causing excess morbidity, some infants with an oxygen index greater than 40 are still referred late for ECMO, after high pressure ventilation has already caused major lung damage, or after other organs - in particular the brainhave been subjected to prolonged hypoxia.

At a time when so little of our neonatal, or indeed paediatric, intensive care practice can be truly described as evidence based, neonatal intensivists will welcome the opportunity to clinically apply the compelling evidence that supports the role of ECMO in the treatment of MAS. This means early recognition of the potential need for ECMO in affected infants, guided by oxygenation index, and early discussion of these cases with designated neonatal ECMO centres. Thus the use of ECMO in severe MAS should be regarded not as a "last resort" when other treatment has failed, but more as an extension of conventional treatment. P J DAVIS

Heartlink ECMO Centre, Glenfield Hospital, Leicester, UK

L S SHEKERDEMIAN

Cardiac Intensive Care Unit, Great Ormond Street Hospital, London, UK LaraShekerdemian@compuserve.com

1 Coltart TM, Byrne DL, Bates SA. Meconium aspiration syndrome: a 6 year retrospective study. Br 7 Obstet Gynaecol 1989;96:411-14.

2 Wiswell TE, Tuggle JM, Turner BS. Meconium aspiration syndrome. Have we made a difference? Pediatrics 1990;85:715-21.

3 Gregory GA, Gooding CA, Phibbs RH, Tolley WH. Meconium aspiration in infants: a prospective study. $\mathcal{F}$ Pediatr 1974;85:848-52.

4 Linder N, Aranda JV, Tsur M, et al. Need for endotracheal intubation and suction in meconium-stained neonates. F Pediatr 1988;112:613-15.

5 Mercier JC, Lacaze T, Storme L, et al. Disease-related response to inhaled nitric oxide in newborns with severe hypoxaemic respiratory failure. French Paediatric Study Group of Inhaled NO. Eur $\not{f}$ Pediat 1998;157:747-52

6 Bartlett RH, Gazzinga AB, Huxtable RF, et al. Extracorporeal circulation (ECMO) in neonatal respiratory failure. $\mathcal{F}$ Thorac Cardiovasc Surg 1977;74:826-33.

7 Lillehei CW, O'Rourke PP, Vacanti JP, Crone RK. Role of extracorporeal membrane oxygenation in selected pediatric respiratory problems. $\mathcal{F}$ Thorac Cardiovasc Surg 1989;98:968-70.

8 Durand M, Snyder JR, Gangitano E, Wu PY. Oxygenation index in patients with meconium aspiration: conventional and extracorporeal membrane with meconium aspiration: conventional and extraco

9 Stolar CJ, Snedelor SM, Bartlett RH. Extracorporeal membrane oxygenation and respiratory failure: experience from the Extracorporeal Life Support Organisation. $\mathcal{F}$ Pediatr Surg 1991;26:563-71.

10 O'Rourke PP, Crone RK, Vacanti JP, et al. Extracorporeal membrane oxygenation and conventional medical therapy in neonates with persistent pulmonary hypertension of the newborn: a prospective randomised study. Pediatrics 1989;84:957-63.

11 Bartlett RH. Extracorporeal life support for cardiopulmonary failure. Curr Probl Surg 1990;27:621-705.

12 Bifano EM, Hakanson DO, Hingre RV, Gross SJ. Prospective randomized controlled trial of conventional treatment or transport for ECMO in infants with persistent pulmonary hypertension (PPHN) [abstract]. Pediatr Res 1992;31:196A.

13 Gross SJ, Bifano EM, Gugenio D, Hakanson DO, Hingre RV. Prospective randomized controlled trial of conventional treatment or transport for ECMO in infants with severe persistent pulmonary hypertension (PPHN): two year follow-up [abstract]. Pediatr Res 1994;36:17A

14 UK Collaborative ECMO Trial Group. UK collaborative randomised trial of neonatal extracorporeal membrane oxygenation. Lancet 1996;341:7582 .

15 Delius R, Anderson H, Schumacher R, et al. Venovenous compares favourably with venoarterial access for extracorporeal membrane oxygenation in neonatal respiratory failure. $\mathcal{F}$ Thorac Cardiovasc Surg 1993;106:329-38.

16 Andrews AF, Klein MD, Toomasian JM, Roloff DW, Bartlett RH Venovenous extracorporeal membrane oxygenation in neonates with respiratory failure. F Pediatr Surg 1983;18:339-46.

17 Klein MD, Andrews AF, Wesley JR, et al. Venovenous perfusion in ECMO for newborn respiratory insufficiency. Ann Surg 1985;210:520-6.

18 Peek GJ, Firmin RK, Moore HM, Sosnowski AW. Cannulation for neonates for venovenous extracorporeal life support. Ann Thorac Surg

19 Desai SA, Stanley C, Gringlas M, et al. Five-year follow-up of neonates with reconstructed right common carotid arteries after extracorporeal membrane oxygenation. F Pediatr 1999;134:428-33

20 Jacobs JP, Goldman AP, Cullen S, et al. Carotid artery pseudoaneurysm as a complication of ECMO. Ann Vasc Surg 1997;11:630-3.

21 Extracorporeal Life Support Organization. ECLS Registry Report, Fuly 1999. Ann Arbor, MI: Extracorporeal Life Support Organization, 1999.

22 Zwischenberger JB, Nyugen TT, Upp R Jr, et al. Complications of neonatal extracorporeal membrane oxygenation. 7 Thorac Cardiovasc Surg 1994:107:838-49.

23 Walsh-Sukys MC, Bauer RE, Cornell DJ, Friedman HG, Stork EK, Hack $M$. Severe respiratory failure in neonates: mortality and morbidity rates and neurodevelopmental outcome. F Pediatr 1994;125:104-10.

24 UK Collaborative ECMO Group. The UK collaborative ECMO trial: follow-up to 1 year of age. Pediatrics 1998;101: E1.

25 Khammash H, Perlman M, Wojtulewicz J, Dunn M. Surfactant therapy in full-term neonates with severe respiratory failure. Pediatrics 1993;92:135-

26 Halliday HL, Speer CP, Robertson B. Treatment of severe meconium aspiration syndrome with porcine surfactant. Collaborative Surfactant Study Group. Eur F Pediatr 1996;155:1047-51.

27 Findlay RD, Taeusch HW, Walther FJ. Surfactant replacement therapy for meconium aspiration syndrome. Pediatrics 1996;97:48-52.

28 The Neonatal Inhaled Nitric Oxide Study Group. Inhaled nitric oxide in full-term and nearly full-term infants with hypoxic respiratory failure. $N$ Engl f Med 1997;336:597-604

29 Rais-Bahrami K, Rivera O, Seale WR, Short BL. Effect of nitric oxide in meconium aspiration syndrome after treatment with surfactant. Crit Care Med 1997;25:1744-7.

30 Foust R, Tran NN, Cox C, et al. Liquid assisted ventilation: an alternative ventilatory strategy for acute meconium aspiration injury. Pediatr Pulmonol 1996;21:316-22.

31 Lotze A, Knight GR, Martin GR, et al. Improved pulmonary outcome after exogenous surfactant for respiratory failure in term infants requiring extracorporeal membrane oxygenation. F Pediatr 1993;122:261-8.

\section{STAMPS IN NEONATOLOGY}

\section{Hospitals and clinics}

Hospitals feature occasionally on stamps but maternity hospitals much less frequently. The maternity hospital in Dakar, Senegal is found on seven stamps from the former French colonies issue in 1942 for the Colonial Child Welfare Fund. The same design is shared-only the country's name altered between stamps. In this instance the stamp from the Ivory Coast is shown.

M K DAVIES

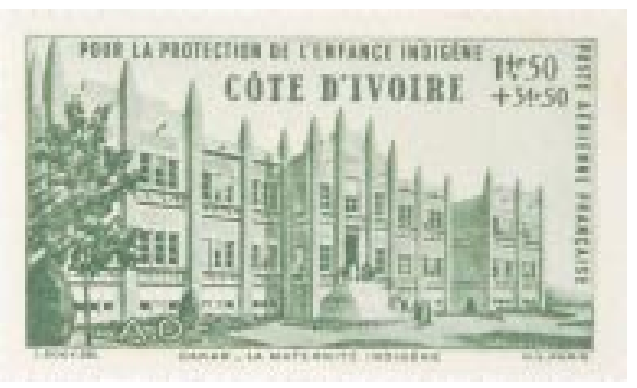

